\title{
Playing it safe
}

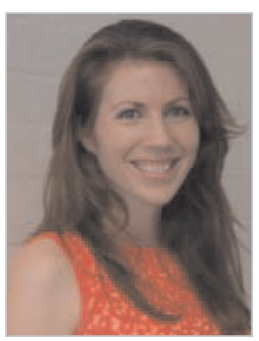

Christine Gee Deputy Editor mja@mja.com.au

doi: 10.5694/mjal4.c1103 improving patient outcomes.

New research from the Concord Medical Emergency Team (MET) Incidents Study (page 528) suggests that the disruption caused by staff leaving normal duties to attend MET calls did not result in major harm to other patients. In their single-centre study, MET call participants were interviewed to ascertain the rate of adverse events and incidents related to their diversion to cover MET calls. After 2663 MET call attendances, participants reported no adverse events and an incident rate of 213.7 per 1000

MET participant attendances, all of which were classified as "minor" or "minimum". Although it is unclear if these findings can be extrapolated to other centres, they certainly provide some reassurance.

While patient safety is clearly top of mind, what about that of health care workers? Knott (page 550) candidly shares his experience of the aftermath of an adverse iatrogenic event that resulted in a patient's death. He observes that in these situations, it is not uncommon for staff involved to be distressed afterwards and so become "second victims" of the event. He describes the lessons learnt from this particular instance and how a patient-centred approach was important to his own recovery process. Hills and Joyce (page 535) report on their exploratory, cross-sectional study of workplace aggression in Australian clinical practice, conducted as part of the third wave of the Medicine in Australia: Balancing Employment and Life survey. Of the responding clinicians, $27.2 \%$ reported experiencing internal aggression (ie, from co-workers) at work, while $67.6 \%$ reported experiencing aggression from external sources (eg, from patients) at work in the preceding 12 months. Adjusted and unadjusted analyses found both of these forms of aggression to be consistently negatively associated with job satisfaction, general life satisfaction and self-rated health. It was suggested that, in turn, such negative effects on clinicians' wellbeing are potentially harmful to the quality and safety of patient care.

Hospitals clearly are a challenging environment for workers' and patients' safety, but safety in the community is also important, especially as outdoor recreational activities increase alongside the seasonal temperature. Howden and colleagues (page 532) report their novel study, which attempted to measure the rate of surfboard-related eye injuries in New South Wales over a 1-year period. Ten cases were reported by ophthalmologists, two of which were described as severe. The authors acknowledge that this was likely to have underrepresented the true rate of injury, and they advocate for the development of safetyenhancing modifications to surfboards. Protective eyewear, although unpopular with surfers, was also suggested as worthy of investigation.

While a beach full of goggle-clad surfers may be hard to envisage this summer, it is encouraging to hear of the efforts underway to make our hospitals and communities safer places to be.

\section{RACGP honours the cream of the crop}

\section{Cate Swannell Careers Editor cswannell@mja.com.au}

Careers follows $p 554$
Dr Michael Crampton was named as the Royal Australian College of General Practitioners' GP of the Year at the College's annual conference (GP14) in Adelaide last month. With interests in e-health and integrated care, and deep involvement in medical education, Dr Crampton talks about his optimism for general practice and the future of primary care (page C1). We also have a round-up of all the award-winners from GP14 (page C2).

\author{
Also in the Careers section is \\ our events calendar for November \\ and December, which includes the \\ $M J A^{\prime}$ 's own continuing professional \\ development events - "An adult \\ respiratory program for general \\ practitioners and non-respiratory \\ specialists" - in Sydney on 29 \\ November, and in Melbourne on \\ 6 December. For more details visit \\ www.doctorportal.com.au/events and \\ page 531 in this issue of the MJA.
}

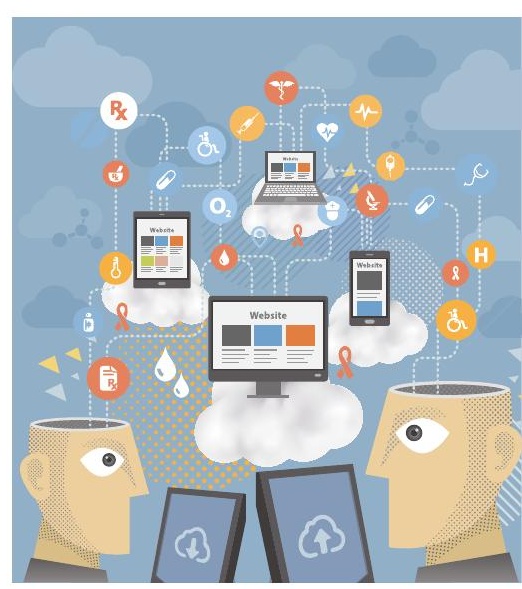

